\title{
Learning Contextualized Music Semantics from Tags Via a Siamese Neural Network
}

\author{
UBAI SANDOUK and KE CHEN, The University of Manchester
}

\begin{abstract}
Music information retrieval faces a challenge in modeling contextualized musical concepts formulated by a set of co-occurring tags. In this article, we investigate the suitability of our recently proposed approach based on a Siamese neural network in fighting off this challenge. By means of tag features and probabilistic topic models, the network captures contextualized semantics from tags via unsupervised learning. This leads to a distributed semantics space and a potential solution to the out of vocabulary problem, which has yet to be sufficiently addressed. We explore the nature of the resultant music-based semantics and address computational needs. We conduct experiments on three public music tag collections—namely, CAL500, MagTag5K and Million Song Dataset-and compare our approach to a number of state-of-the-art semantics learning approaches. Comparative results suggest that this approach outperforms previous approaches in terms of semantic priming and music tag completion.
\end{abstract}

CCS Concepts: - Information systems $\rightarrow$ Multimedia and multimodal retrieval; $\bullet$ Computing methodologies $\rightarrow$ Unsupervised learning; Neural networks; Learning latent representations;

Additional Key Words and Phrases: Distributed semantics learning, tag representation, contextualized concept modeling, out of vocabulary, semantic priming, million song dataset

ACM Reference Format:

Ubai Sandouk and Ke Chen. 2016. Learning contextualized music semantics from tags via a siamese neural network. ACM Trans. Intell. Syst. Technol. 8, 2, Article 24 (October 2016), 21 pages.

DOI: http://dx.doi.org/10.1145/2953886

\section{INTRODUCTION}

Music information retrieval (MIR) is becoming increasingly necessary with the rising rates of music production and demand [Serra et al. 2013]. Manual classification of music is infeasible due to costs, biases, and contradictions introduced by individual experts. As a result, automatic music understanding is vital for providing viable services. Unfortunately, there exists a gap between what machines extract from music and the corresponding human-level understanding, which is well known as the semantic gap [Smeulders et al. 2000]. Efforts to bridge this gap include engineering features [Lew et al. 2006], modeling users' behavior [Schedl et al. 2013], and using concept semantics. Aside from introducing expert knowledge as ontologies [Kim et al. 2008], semantics learning has been dominated by music annotations or tags [Bertin-Mahieux et al. 2010]. Music tracks are associated with textual tags to convey human interpretable concepts describing these tracks. Music understanding is often reduced to automatic annotation of music with suitable tags. Thanks to crowd-sourced [Turnbull et al. 2008] and game-based tagging [Law et al. 2007], large collections of tagging

Authors' addresses: U. Sandouk and K. Chen, School of Computer Science, University of Manchester, Manchester, M13 9PL, UK; emails: \{ubai.sandouk, chen\}@cs.manchester.ac.uk.

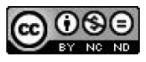

This work is licensed under a Creative Commons

Attribution-NonCommercial-NoDerivs International 4.0 License.

2016 Copyright is held by the owner/author(s).

ACM 2157-6904/2016/10-ART24 \$15.00

DOI: http://dx.doi.org/10.1145/2953886 
information are accessible to music research. The overall quality of the learned semantics is determined by the quality of the annotation and how they are used.

Traditionally, mapping music to tags is realized via multi-label classification. For each tag, a dedicated binary classifier is used in order to predict its appropriateness to a track [Turnbull et al. 2007]. This approach loses tag correlation information concerning much of the intention of any tag's use [Sordo 2011, p.34]. Additionally, this approach depends on analyzing the musical content, which may vary radically when conveying similar concepts. It is particularly difficult to extend this approach to different MIR tasks and applications in the presence of out of vocabulary (OOV) tags. OOV tags refer to those tags that appear in application but are not observed during semantics learning. Generalizing semantics to new tasks as well as new tags is vital for music understanding. Given that the majority of the musical concepts can be represented in tag form, we believe that proper semantics can be learned solely from the analysis of co-occurring tags describing music tracks. In this case, intention and meaning of tags are not affected by the musical content, manually defined ontologies and dictionaries or any other information source.

Semanitcs analysis of tag collections can be done in different ways. Levy and Sandler [2008] suggest the use of semantically coherent methods to obtain tag representations, for example, Latent Semantic Indexing (LSI) [Deerwester et al. 1990] and Probabilistic Latent Semantic Analysis (PLSA) [Hofmann 1999]. These methods uncover statistics that govern the collective use of tags. LSI produces one unique representation for each tag regardless of how it is used in different tracks. We refer to this type of semantics as global relatedness semantics. Another global relatedness model is obtained via analyzing the tag collection using aggregation [Markines et al. 2009] followed by Principle Component Analysis (PCA). Conversely, PLSA produces coherent representations for groups of tags and produces multiple representations for each tag encoded within a representation of a group of tags. We refer to this type of semantics as contextual relatedness. Another contextual relatedness model is obtained via Latent Dirichlet Allocation (LDA) [Blei et al. 2003], which produces a compact representation of a group of tags encoded as activation levels of a set of latent topics. Alternatively, each group of tags can be represented as a vector of binary tag-relevance indicators known as Bag-of-Words (BoW) [Harris 1954]. The BoW can be smoothed by means of Conditional Restricted Boltzmann Machines (CRBM), which results in a tag-based track representation. The smoothed representation captures correlations among tags and is also contextualized by the CRBM's condition information [Mandel et al. 2011], where the condition information is the one-hot representation of the training document carried out by one "activated" unit corresponding to the ID of the document being used for training. Evidently, such condition information cannot be applied to new documents in application.

While the aforementioned methods are able to capture contextual track-to-tag relatedness, they struggle in capturing underlying tag-to-tag relatedness; leading to inconsistent semantics and difficulty across different MIR tasks. For instance, LDA provides a method for relating topics to tags but does not have a clear measure on relatedness among tags. Additionally, these approaches assume a close-set scenario; that is, all tags have to be known during training and used according to their predefined meanings. In reality, different tags may be used to mean the same musical concept, for example, "drums"/ "drumset"; and the same tag may be used to describe more than one musical concept, for example, "guitar" to mean different guitar types. In general, the intended meaning of a single tag cannot be revealed unless all other tags used with it for describing a track are examined. Hereinafter, we use companion tags to refer to these co-occurring tags in an annotation of a track. Furthermore, new tags beyond a predefined vocabulary may also be used by annotators, that is, OOV tags. In summary, 
the existing models fail to yield the proper contextualized relatedness semantics between individual tags and, in particular, there appear OOV tags in applications.

In contrast to the slow progress in tag semantics modeling, natural language processing benefits from a class of distributed language models capturing underlying relatedness among words [Mikolov et al. 2013]. Thanks to their simplicity and capacity in providing generic semantics for numerous linguistic tasks, such models yield semantic spaces where words are embedded based on their syntactic similarity. A distance measure is often used within the semantic space to reflect syntactic relatedness of word pairs. Unlike the dictionary-based models [Iacobacci et al. 2015], distributed language models are trained using words in a corpus without considering any manually constructed information sources, for example, dictionary. Such success inspires further research into distributed semantics. Unfortunately, applying linguistic models directly to tag collections is unreasonable due to the lack of syntactic structure among tags. Without the ordering information, these models can only capture global relatedness neglecting contextualized meanings underlying tags uses. Furthermore, these linguistic models falsely assume linguistic properties of tags as same as those of words. However, tags are beyond words as music tags may be symbols, abbreviations and phrases, for example, "r'n'b", "80s" and "rhythmic loops."

To facilitate our presentation, a document hereinafter refers to a set of co-occurring tags, that is, $\delta=\left\{\tau_{i}\right\}_{i=1}^{m}$, used to describe a music track. For any tag $\tau_{i}, \delta$ forms its local context used to disambiguate the intention of using $\tau_{i}$ for that track. As a result, the specific meaning of a tag, dubbed a concept, is only identified after examining the tag and its local context. In our recent work, we formulate the concept embedding, $\boldsymbol{C E}\left(\tau_{i}, \delta\right)$, problem such that the distance between different concepts embedding reflects contextualized relatedness [Sandouk and Chen 2016]. To solve this problem, we proposed a contextualized semantic learning approach by means of Siamese architecture [Bromley et al. 1993]. By using unsupervised learning, the Siamese neural network establishes a semantics space that embeds concepts properly reflecting their relatedness as Euclidean distance. The space contains multiple representations for each tag in different contexts so that it co-locates with other tags that share the same intention and can estimate the concept underlying an OOV tag from its local context. In this article, we investigate the suitability of this approach in learning musical semantics based on music tag collections. The main contributions in this work are summarized:

-We thoroughly investigate the suitability of Siamese CE approach [Sandouk and Chen 2016] in modeling the musical concepts, including the proper capacity of the network in learning musical semantics, highlighting the emergent structure of the musical tag space and its smooth nature, and assessing the computational efficiency of the network in music domain.

- To make a state-of-the-art contextualized semantic learning model comparable to ours, we propose an improved version for the CRBM model [Mandel et al. 2011] so that it can capture statistical co-occurrence likelihoods of tags as our Siamese architecture does. The semantics learned by the improved CRBM model is significantly different from that done by its original version.

- As the learned CE model can be generalized to tags never seen during training, we examine this nontrivial issue by applying it to the million song dataset, a large dataset facing the long tail problem [Bertin-Mahieux et al. 2011].

-We conduct a thorough evaluation on the learned contextualized semantics based on two benchmark MIR tasks, that is, semantic priming and tag completion, via a comparative study to several state-of-the-art semantic learning models.

The articles is organized as follows: Section 2 reviews the CE model and achieving concepts embedding for tags. Sections 3 and 4 report experimental results in the 
semantic priming and the tag completion tasks, respectively. Section 5 discusses relevant issues, while Section 6 concludes with future works related to this research, including possible concrete uses of CE semantics in MIR tasks.

\section{MODEL DESCRIPTION}

For self-containment, we review our recently proposed approach for learning contextualized semantics from tags [Sandouk and Chen 2016]. As the underpinning techniques, this model is applied to learning semantics from musical tags.

\subsection{Tag and Context Features}

Tags are represented via aggregation [Markines et al. 2009], which captures their global relatedness over the entire training set. Each tag use is weighted using $t$ fidf [Singhal 2001]. Given one training tag $\tau \in \Gamma$ and one training document $\delta \in \Delta$ where $\Gamma$ is the vocabulary and $\Delta$ is the document collection: their binary relatedness is given

$$
t f(\tau, \delta)=\left\{\begin{array}{l}
1 \text { when } \tau \text { appears in } \delta \\
0 \quad \text { otherwise }
\end{array}\right.
$$

The rarity of a tag is considered using inverted document frequency

$$
i d f(\tau)=\log \left(\frac{|\Delta|}{1+|\{\delta \in \Delta: t f(\tau, \delta)=1\}|}\right) \text {, where }\|\cdot\| \text { is the cardinality of a set. }
$$

The $t$ fidf weight is defined by the product of the binary relatedness and the inverted document frequency as $\operatorname{tfidf}(\tau, \delta)=t f(\tau, \delta) \times i d f(\tau)$.

Finally, each tag is described by its usage pattern across all training documents as $\boldsymbol{u}(\tau)=\left\{t f i d f\left(\tau, \delta_{i}\right)\right\}_{i=1}^{|\Delta|}$. Consequently, global relatedness between two training tags $\tau_{1}, \tau_{2} \in \Gamma$ is measured by the dot product of their respective usage vectors $T\left(\tau_{1}, \tau_{2}\right)=<$ $\boldsymbol{u}\left(\tau_{1}\right), \boldsymbol{u}\left(\tau_{2}\right)>$, which leads to tag representation of $|\Gamma|$ features as

$$
\boldsymbol{t}(\tau)=\left\{T\left(\tau, \tau_{i}\right)\right\}_{i=1}^{|\Gamma|} .
$$

Local contexts are captured via LDA [Blei et al. 2003] over all tags in a document. LDA assumes a set of latent independent topics $\Phi$ that softly cluster the documents based on the used tags resulting in a probabilistic topic model (PTM) representation of the entire document. During training, the process estimates scalar priors B for the Dirichlet distributions to model the tags within each topic as well as the scalar prior $\mathrm{B}^{0}$ in the Dirichlet distribution used to model the topics. After training, the probability of a tag $\tau \in \Gamma$ is subject to $p(\tau \mid \phi) \sim$ Categorical(Dirichlet $(\mathrm{B}))$ given a topic $\phi \in \Phi$ where $p(\phi) \sim \operatorname{Dirichlet}\left(\mathrm{B}^{0}\right)$. For a document $\delta, p(\phi \mid \delta) \sim p(\phi) \prod_{\tau \in \delta} p(\tau \mid \phi)$, the local context of a tag is represented by a vector of $|\Phi|$ features corresponding to the $|\Phi|$ topic distribution output with respect to $\delta$ :

$$
\boldsymbol{l}(\delta)=\left\{l_{c}(\delta)\right\}_{c=1}^{|\Phi|}, \quad l_{c}(\delta)=p\left(\phi_{c} \mid \delta\right) .
$$

\subsection{Siamese Architecture}

To learn contextualized semantics, we use the Siamese architecture illustrated in Figure 1. We train a deep neural network that predicts companion tags for a tag based on the input features. Given an example $\boldsymbol{x}_{k}$, consisting of tag $\tau$ and document $\delta$, input features are the result of concatenating the tag and context features: $\boldsymbol{x}_{k}(\tau, \delta)=\{\boldsymbol{t}(\tau), \boldsymbol{l}(\delta)\}$. The network consists of $H$ hidden layers where a layer $h$ is characterized by weight $W_{h}$ and bias $b_{h}$ parameters. The output of layer $h$, for example $\boldsymbol{x}_{k}$, is

$$
\boldsymbol{z}_{h}\left(\boldsymbol{x}_{\boldsymbol{k}}\right)=\boldsymbol{f}\left(W_{h} \cdot \boldsymbol{z}_{h-1}\left(\boldsymbol{x}_{k}\right)+\boldsymbol{b}_{h}\right), 1 \leq h \leq H,
$$




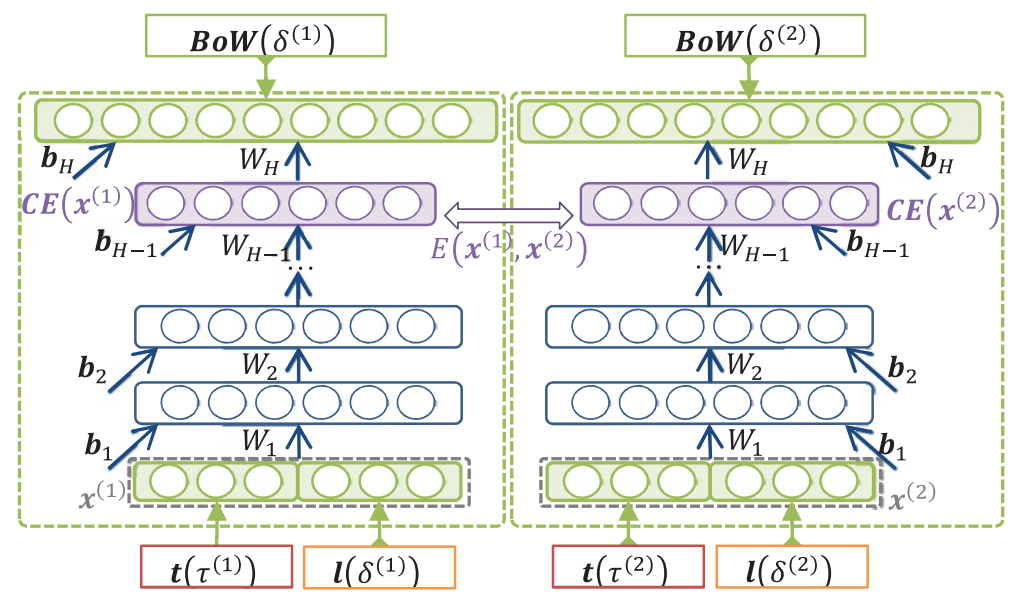

Fig. 1. The Siamese network for contextualized music semantics learning (see Section 2.2 for notation).

where $\boldsymbol{f}($.$) is the element-wise hyperbolic tangent function. The output of the (H-1)^{\text {th }}$ hidden layer, that is, the penultimate layer, is used as the semantic representation. We refer to this representation as contextual embedding (CE). We stipulate the raw input features are $\boldsymbol{x}=\boldsymbol{z}_{0}(\boldsymbol{x})$, the contextualized embedding is $\boldsymbol{C E}(\boldsymbol{x})=\boldsymbol{z}_{H-1}(\boldsymbol{x})$ and the prediction is $\hat{\boldsymbol{y}}(\boldsymbol{x})=\boldsymbol{z}_{H}(\boldsymbol{x})$. Lastly, for a pair of inputs to the Siamese network $\boldsymbol{x}^{(1)}=\left\{\boldsymbol{t}\left(\tau^{(1)}\right), \boldsymbol{l}\left(\delta^{(1)}\right)\right\}$ and $\boldsymbol{x}^{(2)}=\left\{\boldsymbol{t}\left(\tau^{(2)}\right), \boldsymbol{l}\left(\delta^{(2)}\right)\right\}$, the embedding similarity is defined by the Euclidean distance between their contextual embedding:

$$
\mathrm{E}\left(\boldsymbol{x}^{(1)}, \boldsymbol{x}^{(2)}\right)=\mathbf{C E}\left(\boldsymbol{x}^{(1)}\right)-\mathbf{C E}\left(\boldsymbol{x}^{(2)}\right)_{2} .
$$

Here in after, we shall drop all explicit parameters to simplify presentation, for example, $y_{k}$ stands for $\boldsymbol{y}_{k}\left(\boldsymbol{x}_{k}(\tau, \delta)\right)$ and $y_{k j}$ means the $j^{t h}$ entry of $y_{k}$.

\subsection{Learning Algorithm}

For each training document $\delta$ of $m$ tags, we create $m$ positive training examples where each example consists of one focused tag and the shared local context features. The prediction targets for these examples are the BoW of $\delta$. Moreover, we artificially synthesize $m$ negative training examples where each example consists of one focused tag that does not appear in $\delta$ and the local context features of $\delta$. The prediction targets for these examples are the complement of $\mathrm{BoW}$ of $\delta$. These examples reduce the domination of context features in predicting the related tags.

The deep network is pretrained with the greedy layer-wise initialization with sparse autoencoders suggested in Bengio et al. [2007]. The initialization is followed by the error back propagation training to predict the target representation from the tag and context input. The loss is a variant of the cross-entropy cost. Given the entire training dataset $X=\left\{x_{k}(\tau, \delta)\right\}_{k=1}^{K}$, the loss is thus defined by

$$
\mathcal{L}_{P}(X ; \Theta)=-\frac{1}{2 K|\Gamma|} \sum_{k=1}^{K} \sum_{j=1}^{|\Gamma|}\left(\kappa_{k}\left(1+y_{k j}\right) \log \left(1+\hat{y}_{k j}\right)+\left(1-\kappa_{k}\right)\left(1-y_{k j}\right) \log \left(1-\hat{y}_{k j}\right)\right) .
$$

Here, $\kappa_{k}=\frac{\left|\left\{i: y_{k i}=1\right\}_{i=1}^{|\Gamma|}\right|}{|\Gamma|}$ is a weight that highlights the cost of a false-negative error. This optimization is carried out via the Stochastic Back Propagation (SBP) [Bottou 2012]. After completing the learning of this network, we obtain the initial embedding. 
We train the Siamese architecture by coupling two copies of the initial network operating together for distance learning. Given an input examples pair $\boldsymbol{x}^{(1)}$ and $\boldsymbol{x}^{(2)}$, the contextualized semantic similarity is reflected by the Kullback-Leibler (KL) divergence of their respective contexts:

$$
K L\left(x^{(1)}, x^{(2)}\right)=\sum_{c=1}^{|\Phi|}\left(\left(l_{c}^{(1)}-l_{c}^{(2)}\right) \log \left(\frac{l_{c}^{(1)}}{l_{c}^{(2)}}\right)\right) .
$$

Moreover, the distance learning makes use of the semantic similarity to penalize the embedding if distances deviate away from a target distance. Given a pair of input examples $\boldsymbol{x}_{n}^{(1)}$ and $\boldsymbol{x}_{n}^{(2)}$, we stipulate $\mathbb{E}=E\left(\boldsymbol{x}_{n}^{(1)}, \boldsymbol{x}_{n}^{(2)}\right)$ and $\mathbb{S}=e^{\frac{-\lambda}{2}\left(K L\left(\boldsymbol{x}_{n}^{(1)}, \boldsymbol{x}_{n}^{(2)}\right)\right)}$, where $\lambda$ is a sensitivity parameter that affects the degree to which the embedding is dominated by context similarity. Given two subsets $X^{(1)}$ and $X^{(2)}$ of cardinality $N$ of randomly selected examples via pairing from $X$, the following loss is used for Siamese learning:

$$
\mathcal{L}_{S}\left(X^{(1)}, X^{(2)} ; \Theta\right)=\frac{1}{N} \sum_{n=1}^{N}\left(I_{1}(\mathbb{E}-\beta(1-\mathbb{S}))^{2}+I_{2} \rho(\mathbb{E}-\beta(1-\mathbb{S}))^{2}+I_{3}(\mathbb{E}-\beta)^{2} \mathbb{S}\right),
$$

where $\sum_{k=1}^{3} I_{k}=1 ; I_{k} \in\{0,1\}$ are indicators which distinguish three possible cases of input pairs: $I_{1}=1$ when both inputs are positive examples; $I_{2}=1$ when both inputs are negative examples; and $I_{3}=1$ when one input is a positive example and the other is a negative one. $\beta$ is a scaling factor used to ensure tags spread over the embedding space and $0 \leq \rho<1$ is an importance factor which reduces the effects of case $I_{2}=1$.

During distance learning, each component network is also trained simultaneously to predict the BoW of its input to avoid an abrupt change of the CE learned initially with a single network. Thus, the distance learning needs to combine two loss functions defined in Equations (3) and (4), which leads to a multi-objective loss:

$$
\mathcal{L}\left(X^{(1)}, X^{(2)} ; \Theta\right)=\sum_{i=1}^{2} \mathcal{L}_{P}\left(X^{(i)} ; \Theta\right)+\alpha \mathcal{L}_{S}\left(X^{(1)}, X^{(2)} ; \Theta\right),
$$

where $\alpha$ is a trade-off parameter that reconciles two different losses.

The optimization problem defined in Equation (5) is solved via SBP. Iteratively, small batches of examples are randomly selected, their loss measured, and the parameters updated accordingly. The networks are kept identical by averaging the parameters after each update. Training continues until validation $P @ 2$ (c.f. Section 3.5.) stops improving. See the appendix of Sandouk and Chen [2016] for further details on learning algorithm.

\subsection{Tag Contextual Embedding}

For an input tag $\boldsymbol{x}(\tau, \delta)=\{\boldsymbol{t}(\tau), \boldsymbol{l}(\delta)\}$, an embedding network is used to obtain the CE representation denoted by $\boldsymbol{C E}(\tau, \delta)$. For the two-stage learning procedure, the model generates two $\boldsymbol{C E}(\tau, \delta)$ representations by using the network trained with only the prediction loss in Equation (3) or using the multi-objective loss in Equation (5).

For an OOV tag $\tau_{\text {oov }}$ appearing in a document $\delta$ alongside in-vocabulary tags, that is, $\delta=\tau_{\text {oov }} \cap \delta_{i v} ; \delta_{i v}=\left\{\tau_{i}\right\}_{i=1}^{\left|\delta_{i v}\right|}$, we use CE representations of all $\left|\delta_{i v}\right|$ companion tags denoted as $\left\{\boldsymbol{C E}\left(\tau_{i}, \delta_{i v}\right)\right\}_{i=1}^{\left|\delta_{i v}\right|}$ and estimate an OOV tag representation as the centroid of these vectors $\boldsymbol{C E}\left(\tau_{\text {oov }}, \delta\right)=\frac{1}{\left|\delta_{i v}\right|} \sum_{i=1}^{\left|\delta_{i j}\right|} \boldsymbol{C E}\left(\tau_{i}, \delta_{i v}\right)$. This $\mathrm{CE}$ representation allows the estimation of intentions of OOV tags based on local contexts. 


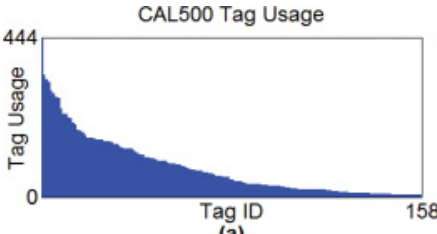

(a)

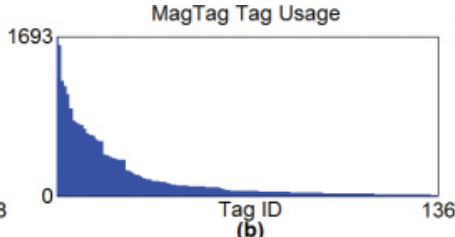

(b)

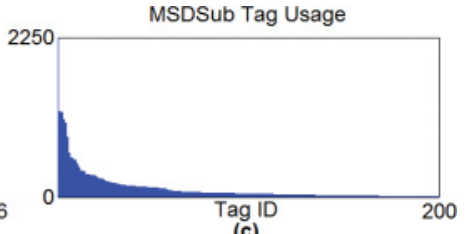

(c)

Fig. 2. Frequency of tags used in different datasets. (a) CAL500. (b) MagTag5K. (c) MSDSub.

\section{EVALUATION OF LEARNED SEMANTICS}

In this section, we first describe the datasets and the settings used in our experiments. Then we demonstrate the contextualized semantics learned by the CE model via visualization. Finally, we report experimental results on semantic priming.

\subsection{Dataset and Feature Extraction}

In our experiments, we employ three publically available datasets: CAL500 [Turnbull et al. 2007], MagTag5K [Marques et al. 2011], and Million Song Dataset (MSD) [BertinMahieux et al. 2011]. These datasets exhibit different tag usage distributions, as shown in Figure 2. In tagging, there exists the so-called long-tail problem where the majority of available tags are rarely used. From Figure 2, it is observed that CAL500 does not suffer from this problem as severely as the other two datasets.

CAL500 is a dataset of 500 songs annotated using 158 unique tags via surveys. The dataset uses tags densely given the fact that there are 25 tags per document, on average. MagTag5K is a controlled version of MagnaTune where repeats and contradictions have been removed. MagnaTune is the result of an online annotation game that allowed users to evaluate the appropriateness of complete tag sets rather than individual tags at a time [Law et al. 2009]. MagTag5K contains 5,259 documents and a vocabulary of 136 tags. It is sparser than CAL500 with five tags per document, on average. MSD is a dataset of 1 million songs' information. Many of the songs are tagged through last.fm, a crowd-sourced annotation website. The original dataset contains a vocabulary of 520,539 tags. In our work, we use the 300 most used tags and the 14,627 documents using only these tags to form a subset, named MSDSub. MSDSub has 3.2 tags per document, on average.

We simulate OOV scenarios by reserving a number of tags away from semantics learning; once a tag is reserved, any relevant documents containing it is never used in training the model. We randomly select and reserve 22 tags and the 1,160 relevant documents in MagTag5K and 100 tags and 7,054 relevant documents in MSDSub. The remaining documents are used for feature extraction where we obtain 114 and 200 tag features for MagTag5K and MSDSub, respectively. However, the high density of CAL500 does not allow for such setting because reserving a single tag would disable around 160 relevant documents while there are only 500 documents in this dataset. Thus, all 158 tags are used in training, which yields 158 tag features. To model local contexts, we empirically sought the proper number of topics in LDA by using the Dirichlet Hierarchical Process suggested in Teh et al. [2006]. As a result, 25, 19, and 23 topics are used for CAL500, MagTag5K, and MSDSub, respectively.

\subsection{Experimental Settings}

In our experiments, we adopt three-fold cross-validation (CV) by randomly splitting a dataset into three subsets of equal sizes. In each fold, two-thirds are used for training the $\mathrm{CE}$ model, and the remaining third is used for validation and testing. As a result, in each fold, there are 40,300, and 500 documents for validation and 127, 1,007, and 3,487 
Table I. Averaging Time (Second) Taken in Different Training Stages and Test

\begin{tabular}{|l|c|c|c|}
\hline \multicolumn{1}{|c|}{ Dataset } & CAL500 & MagTag5K & MSDSub \\
\hline \hline Pre-training (/fold) & 190 & 134 & 177 \\
\hline Prediction training (/fold) & 6,149 & 12,406 & 20,495 \\
\hline Siamese training (/fold) & 3,032 & 3,068 & 3,928 \\
\hline CE representation extraction (/instance) & $2.3 \times 10^{-3}$ & $1.5 \times 10^{-3}$ & $3.3 \times 10^{-3}$ \\
\hline
\end{tabular}

documents for test in CAL500, MagTag5K, and MSDSub, respectively. Determining the hyper-parameters is done via grid search. It is worth stating that the initialization of the deep network is done with sparse auto-encoders in a greedy layer-wise way instead of a random initialization. Consequently, we found a proper network structure: input $\rightarrow 100 \rightarrow 100 \rightarrow 10 \rightarrow$ output, where $\lambda=0.5, \beta=3$, and $\alpha$ values are in the range $[0.1,1]$ for all three datasets.

For thorough evaluation, the CE model is evaluated in two forms: the trained initial semantics (CE) and the fine-tuned Siamese semantics (Siamese CE). Furthermore, we compare our approach to several state-of-the-art approaches reviewed in Section 1, including PLSA [Levy and Sandler 2008], LDA [Law et al. 2010], and CRBM [Mandel et al. 2011]. Unfortunately, CRBM uses a one-hot representation for condition or context. While this condition produces smoothed BoW representations for all the training documents, learned semantics cannot be applied to new documents and across MIR tasks. For proper comparison between CE and CRBM, we come up with an improved version for CRBM by replacing the one-hot condition representation with the same context representation used in CE, that is, PTM. We name this nontrivial extension $C R B M(P T M)$. The architecture and cost function in Mandel et al. [2011] remain the same in CRBM(PTM). It is worth highlighting that the semantics learned with CRBM(PTM) are significantly different from those learned by the original CRBM and the biggest difference appears in those documents of similar PTM context features having maximally dissimilar one-hot context representations. The CRBM model implicitly encodes the "popularity" of tags in its bias vector so that it is more likely to predict popular tags. Among all the models used for comparison, the resultant semantics of $\mathrm{CRBM}(\mathrm{PTM})$ are the closest to those obtained from the CE model. We also include the Random model, which responds with random relatedness values between pairs of tags. All the results reported in this section are based on the test subset in three folds. Such results indicate the generalization ability of the learned semantics. Apart from the goal from a learning perspective, results on training data are also meaningful and can be directly employed in various IR tasks. Due to the limited space, however, we have to report results on training sets in an appendix.

\subsection{Computational Efficiency}

It is well known that training deep neural networks often takes long times and may need the use of GPUs to speed up the training [Raina et al. 2009]. In our experiments, we use a Linux server with $24 \mathrm{CPUs}$ running at $2.0 \mathrm{GHz}$ and memory of 128GB. All our algorithms were implemented in Matlab [2012a].

For three-fold CV, the average training time at different training stages is listed in Table I. Also, the average time in extracting the CE representation of an instance in test is reported. Obviously, the training time generally depends on the number of input features and the size of different datasets. In particular, we observe that the pretraining on CAL500, a dataset of densely used tags, takes longer than that on MagTag5K and MSDSub. Time spent during test for CE representation extraction is similar in the three datasets despite the difference in the number of input features. 


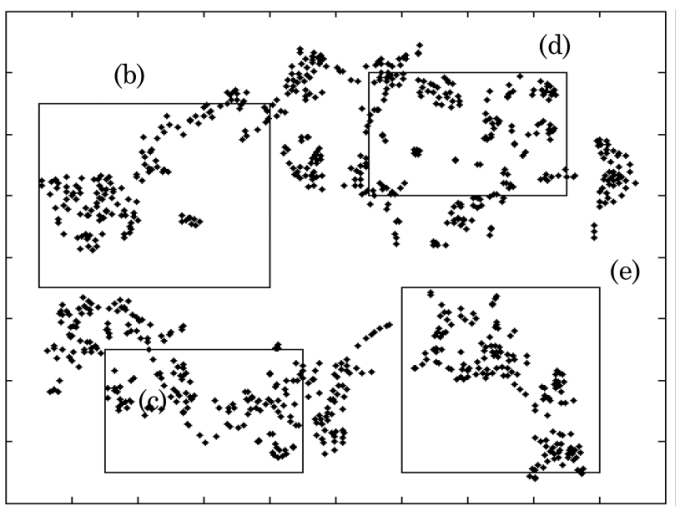

(a)

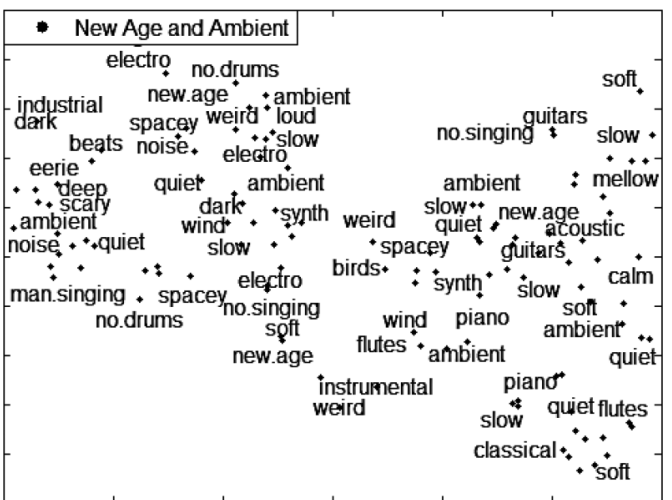

(c)

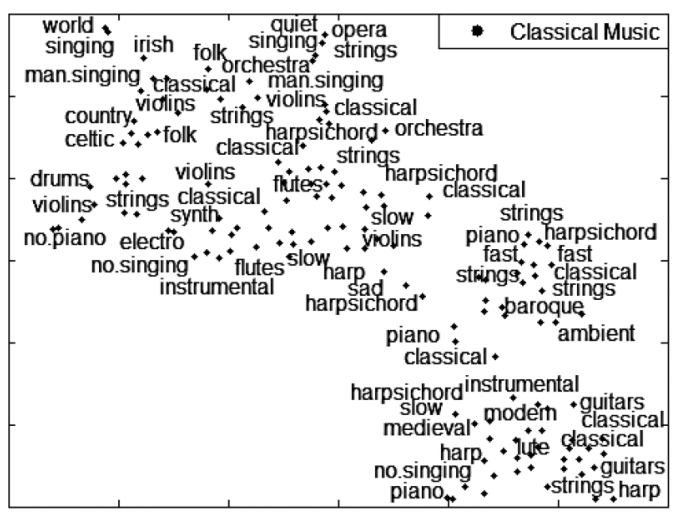

(e)

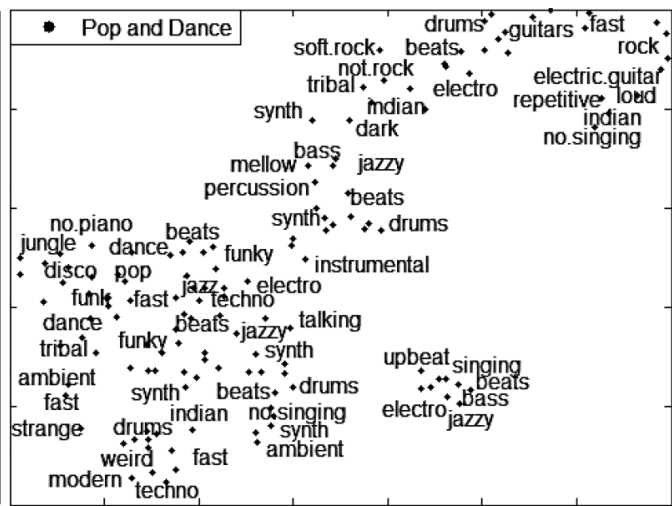

(b)

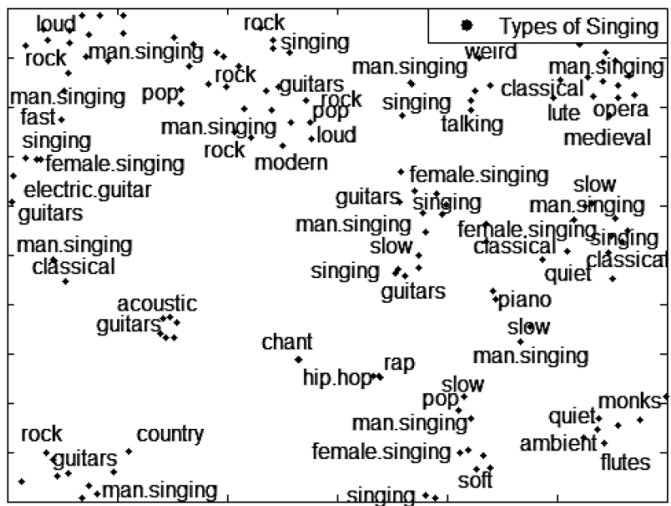

(d)

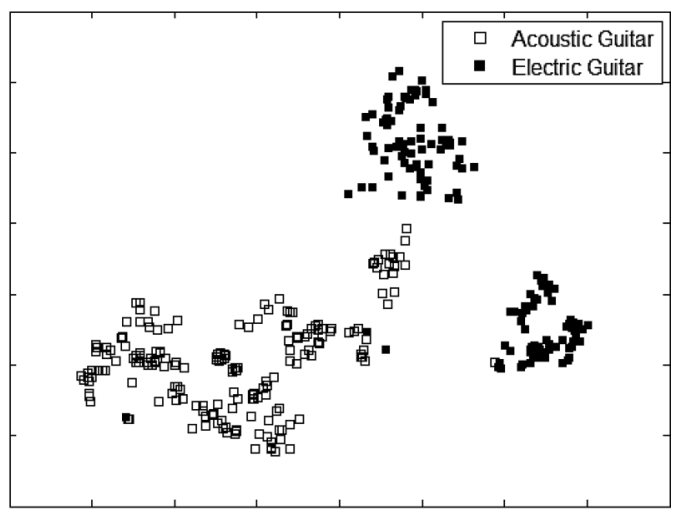

(f)

Fig. 3. 2D projection of CE representations of exemplar tags. (a) All tags in 200 randomly selected documents form MagTag5K. (b)-(e) Annotations of tags inside four boxed regions in (a). (f) All 388 "guitar" tag instances in MagTag5K.

\subsection{Visualization}

By using unsupervised t-SNE [van der Maaten and Hinton 2008], we project the CE representations of all tags used in 200 randomly selected documents from MagTag5K onto a 2D plane for visualization. As illustrated in Figure 3(a), each dot represents a tag 
in one document. To see the space clearly, we annotate dots with their corresponding tags. However, the annotations render the plot overly crowded and difficult to read. Therefore, we highlight only four densely populated regions confined in these boxes in Figure 3(a) and illustrate the details of four regions in Figures 3(b) through 3(e) by zooming in on four regions. Within these plots, close duplicates of any tag were annotated only once for better visual effect. Moreover, we label each region with the captured concepts according to human knowledge. It is observed that four regions in Figures 3(b) through 3(e) correspond to the concepts of "Pop and Dance," "New Age and Ambient," "Types of Singing," and "Classical Music," respectively.

From Figures 3(a) through 3(e), it is observed that there is a swift transition between different musical concepts and the continuous nature of the embedding space is noticeable. For example, there is a clear transition in Figure 3(c) from electrical new age music (up left) to acoustic classical ambient (down right). In Figure 3(e), there is a clear transition from concepts associated with piano-based classical music (down right) through baroque music (middle center) to folk classical music (up left). In addition, transitions between the different regions are swift as well, for example, those tags concerning Rock music (loud, electric, rock, etc.) near the right boundary of the "Pop and Dance" region in Figure 3(b) and those near the left boundary of the "Types of Singing" region in Figure 3(d). A similar effect is also observed between the "Pop and Dance" and the "New Age and Ambient" boundaries in Figures 3(b) and 3(c).

As stated previously, a complete musical concept is often formulated by using a set of coherent tags collectively instead of an individual tag. This has been carried out by co-locating such coherent tags in CE space. For instance, tags "eerie," "scary," "deep," and "ambient" seen on the middle left in Figure 3(c) collectively form a single musical concept corresponding to a specific type of chilling ambient music. Similarly, "harp," "no.singing," and "piano" at the bottom center of Figure 3(e) collectively form another musical concept describing a specific type of classical music. We also observe that many tags are located in multiple regions in order to describe different musical concepts in context. For instance, the tag "slow" has been found simultaneously in Figures 3(c), 3(d), and 3(e) to express slow and quiet types of ambient sounds and music relevant to the "New Age and Ambient," slow songs and singing voice linked to the "Types of Singing," and slow classical music often correlated with the use of violins and flutes pertaining to "Classical Music," respectively.

Figure 3(f) shows a 2D projection of CE representations of all 388 instances of the "guitar" tag found in MagTag5K, where three clusters emerge. According to our genre knowledge, we identify that two clusters marked by "口" are associated with the documents of acoustic nature and the third one marked by " $\square$ " corresponds to the documents of electric nature. This visualization clearly demonstrates the polysemous aspects of a tag in different contexts. Here, we emphasize that our learning model captures different meanings of a tag based on the intention of using this tag rather than mere co-occurrence with other tags.

In summary, the visualization in Figure 3 demonstrates several useful properties that facilitate MIR, including co-located musical concepts, semantic distance within the $\mathrm{CE}$ space reflecting the corresponding contextualized relatedness, swift transition between musical concepts and the polysemous aspects of a tag in different contexts.

\subsection{Semantic Priming}

For evaluation of learned semantics, we use semantic priming, a benchmark IR task, where all semantically related tags are expected to be identified given a query tag [Lund and Burgess 1996]. Successful priming is observed when the model is presented with a query tag in context and the model is able to identify related tags without including any nonrelated ones. This abstract task acts as a generic natural evaluation test bed 
for semantics without favoring any application. In order to complete the evaluation, we need a set of query tags in context along with their ground truth information that associates related tags under that context. However, due to the lack of such groundtruth information, we iteratively use one evaluation document as a coherent set of semantically related tags that should be identified as a response to a query tag. As a result, all tags in an evaluation document $\delta$ are used as ground truth, and each of these tags is used as a query tag in turn. In other words, the quality of semantics learned by different models can be evaluated by examining how well one query tag and its local context can identify its companion tags in the same evaluation document. For such a task, a learning model is assessed by measuring the performance of retrieving the top $k$ tags in response to a query tag. Given $\boldsymbol{r}$, the predicted ordered list of tags, and $\overline{\boldsymbol{r}}_{k}$, the top $k$ tags of $r$, Precision at $k$ is

$$
P @ k(k ; \delta, \boldsymbol{r})=\frac{\left|\delta \cap \overline{\boldsymbol{r}}_{k}\right|}{k},
$$

which indicates the accuracy of the model in correctly identifying $k$ related tags given one query tag. Unfortunately, this measure is affected by the length of an evaluation document such that its values drop quickly when $k$ exceeds the length of the evaluation document, and hence evaluation across multiple documents becomes meaningless. This issue can be solved using Mean Average Precision (MAP): the average $P @ k$ values for a query up to the number of tags in the evaluation document

$$
M A P=\frac{1}{|\delta|} \sum_{i=1}^{|\delta|} P @ k(i ; \delta, \boldsymbol{r})
$$

Intuitively, MAP measures the percentage of the identified tags that are "correct." High MAP results mean that the assessed model identifies relevant tags at the top of the retrieved list or in the low recall range. However, it does not measure the performance over the entire retrieved list. In fact, the performance in the high recall range is often important for tasks such as tag completion and query expansion where all the related tags need to be identified. Therefore, we also evaluate the performance over the entire retrieved list by measuring the numbers of retrieved tags required to achieve the standard 11 recall levels: $0.0,0.1,0.2, \ldots, 1.0$ and their corresponding precision [Manning et al. 2009] with $\operatorname{Recall}(\delta, \overline{\boldsymbol{r}})=\frac{|\delta \cap \overline{\boldsymbol{r}}|}{|\delta|}$ and $\operatorname{Precision}(\delta, \overline{\boldsymbol{r}})=\frac{|\delta \cap \overline{\boldsymbol{r}}|}{|\overline{\boldsymbol{r}}|}$. Finally, we aggregate all the results in a single figure of merit by using the Area Under Curve (AUC). Unlike the Receiver Operating Characteristic (ROC) for binary classification, our used AUC allows the assessment of a ranked list. For two models with similar MAP levels, higher AUC suggests better performance in the high recall range, that is, identifying all the relevant tags. Figures 4, 5, and 6 depict the MAP and the AUC results for different contextualized semantic learning models to be compared on three datasets, CAL500, MagTag5K, and MSDSub, respectively.

Figure 4 shows the semantic priming results on CAL500. As this is a dense dataset, relevant tags are correctly predicted in small $k$ values by most models. For example, the Random model achieves 56\% in P@2 shown in Figure 4(a). Moreover, CRBM(PTM) is comparable to Siamese CE in terms of MAP and has a higher precision in the recall range of $[0.1,0.3]$ because it tends to predict popular tags. The good performance at the high recall range of CE is evident in Figure 4(b) and reciprocally results in a clear advantage in AUC. Overall, Siamese CE outperforms other models with statistical significance (Student's t-test p-value $<0.01$ ). Confidence intervals at $95 \%$ reveal a gain in accuracy when using Siamese CE as indicated by AUC. Moreover, we measure the standardized difference, that is, effect size, between the reported statistics. For our purposes, we report Cohen's d effect size, which measures the standardized difference 


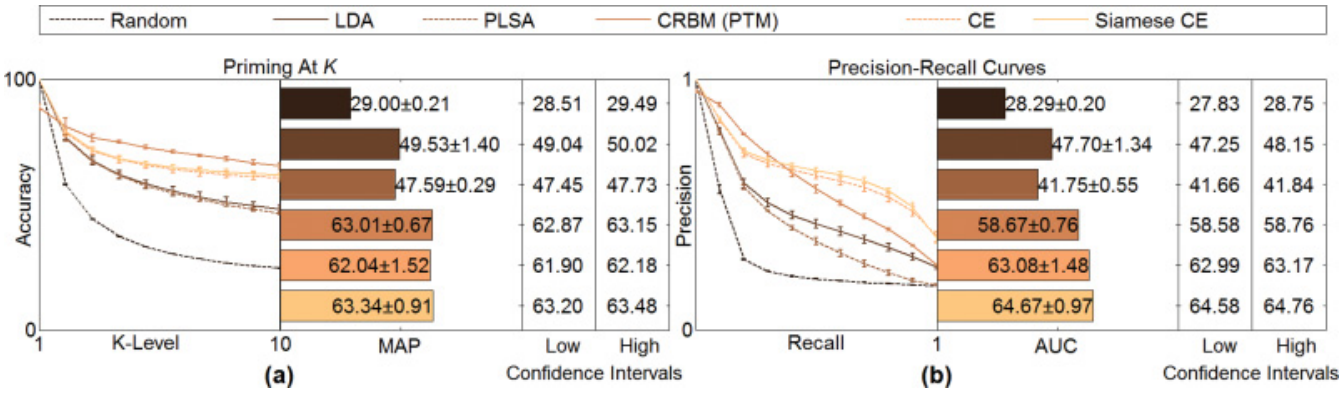

Fig. 4. Semantic priming performance of different models on test subsets of CAL500. (a) The priming accuracy at different $k$ levels as well the MAP mean and standard errors. (b) The Precision-Recall curves as well as the AUC mean and standard errors. This notation is applied to all the figures hereinafter.

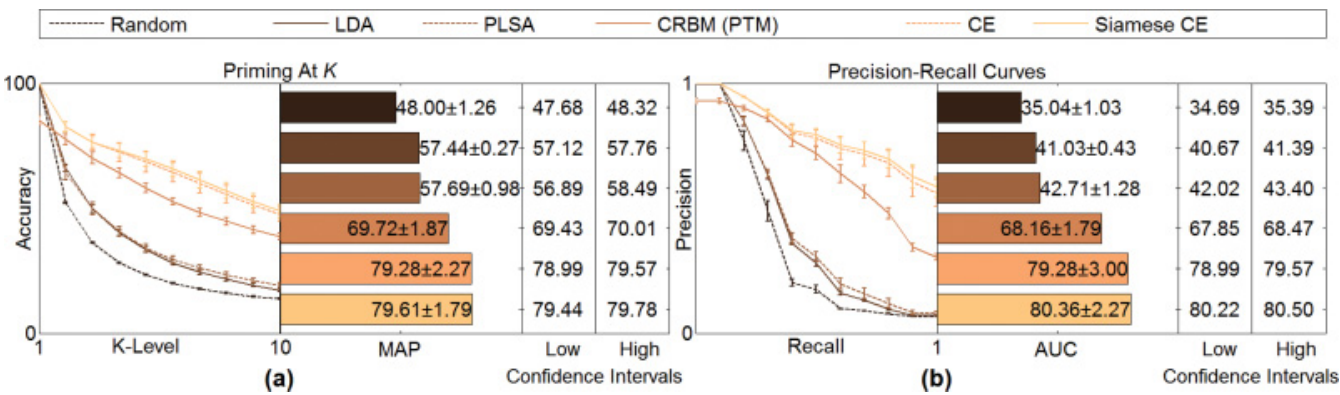

Fig. 5. Semantic priming performance of different models on test subsets of MagTag5K.

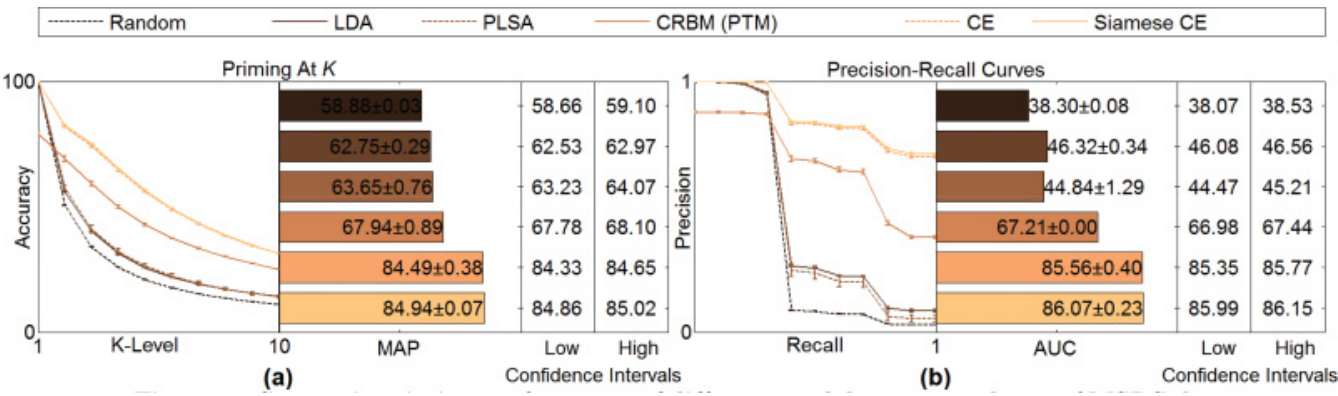

Fig. 6. Semantic priming performance of different models on test subsets of MSDSub.

between two independent samples means [Cohen 1977]. Cohen's d effect size between Siamese CE and CRBM(PTM) is 0.42 in MAP and 7.00 in AUC, and the effect size between Siamese CE and CE is 1.04 in MAP and 1.27 in AUC. These results suggest a clear advantage for Siamese CE over CRBM(PTM) in the low recall range as well as a significant advantage in the high recall range as shown in Figure 4(b). In contrast, it is evident from Figure 4 that the performance of LDA and PLSA is inferior to that of CRBM(PTM) and that of the CE model. It is also observed from Figure 4(b) that all models struggle in the high recall range due to having only 335 training documents. The lack of sufficient training data on this dense dataset inevitably limits the generalization ability of any model.

Figure 5 shows the semantic priming results on MagTag5K. In this case, the CE model performs better than all other models especially in the high recall range with 
statistical significance (Student's t-test $p$-value $<0.01$ ). Short documents in this dataset account for the high MAP accuracy of the Random model. We observe that LDA and PLSA perform similar to each other with little improvement over the Random model. In contrast, CRBM(PTM) performs better than both LDA and PLSA but less significantly than observed on CAL500 due to the sparsity of MagTag5K. We notice that the Siamese $\mathrm{CE}$ outperforms $\mathrm{CE}$ as well as reduces variance of results amongst different folds. The confidence intervals reveal a slight advantage by using Siamese CE in MAP and a significant advantage in AUC. Moreover, the effect size between Siamese CE and $\mathrm{CRBM}(\mathrm{PTM})$ is 5.49 in MAP and 5.56 in AUC, and the effect size between Siamese $\mathrm{CE}$ and $\mathrm{CE}$ is 0.16 in MAP and 0.41 in AUC. It is worth clarifying that the advantage of Siamese CE over CE looks modest in terms of generalization but the nature of this dataset, the difficulty in understanding tags, and the advantage gained by Siamese CE in the high recall range, as evident in Figure 5(b), suggest that Siamese CE may be more suitable to some MIR applications.

Figure 6 shows the semantic priming results on MSDSub. Due to the extremely short document lengths (3.2 tags, on average) in MSDSub, the $P @ k$ drops quickly after $k=3$ for all models. It is seen from Figure 6(a) that both CE and Siamese CE outperform other models with statistical significance (Student's t-test $p$-value $<0.01$ ). In particular, the CE model performs extremely well in the high recall range while LDA and PLSA perform poorly and similarly to the Random model. The deteriorated performance of other models in the high recall range is due to the size of the vocabulary and the sparsity of this dataset, which does not affect the CE model. Although CRBM(PTM) still outperforms LDA and PLSA, it clearly underperforms the CE model as evident in Figure 6. Similar to results on MagTag5K, the Siamese CE slightly outperforms $\mathrm{CE}$ and reduces the variance amongst different folds. The effect size between Siamese $\mathrm{CE}$ and $\mathrm{CRBM}(\mathrm{PTM})$ on MSDSub reaches 26.88 in MAP and 117.64 in AUC, and the effect size between Siamese CE and CE is 1.67 in MAP and 1.54 in AUC. The same conclusion drawn from results in MagTag5K is applicable in this dataset. Hence, the CRBM(PTM), our extension of the CRBM [Mandel et al. 2011], is a good candidate for capturing contextualized relatedness between tags, as it always outperforms LDA and PLSA. Nevertheless, the CE model generally yields statistically significant better results than the CRBM(PTM) on all three datasets according to all four evaluation criteria. In particular, the good performance in the high recall range suggests that semantics learned by the CE model would facilitate auto-annotation or auto-tagging, tag completion and semantic query expansion required by MIR tasks.

The OOV problem is not unique to the CE model. It is encountered whenever the semantics is used in applications where tags' use is not restricted, such as online tagging services, which allow users to tag music using any tag no matter if such tag had been used before. Although the concept conveyed in an OOV tag is less certain than an in-vocabulary tag, it is very important to exploit semantics underlying the OOV tag. To the best of our knowledge, none of the existing semantic learning models address the OOV issue. Fortunately, we can infer the intention of an OOV tag based on the $\mathrm{CE}$ representations of co-occurring companion tags in the document containing the OOV tag. In our experiments, OOV tags are used as query tags for semantic priming and then the primed lists are compared against their ground truth in-vocabulary tags in the evaluation document. To demonstrate the effectiveness of our approach, we also applied the Random model for baseline performance.

Figures 7 and 8 show the priming results on the OOV tags reserved in MagTag5K and MSDSub (c.f. Section 3.1). It is observed that Siamese CE performs slightly better than $\mathrm{CE}$ due to the learned semantic distance reflecting the contextualized relatedness better. By comparing to results on test subsets of in-vocabulary tags shown in Figures 5 and 6, the OOV performance of the CE model is quite close on two datasets, while 


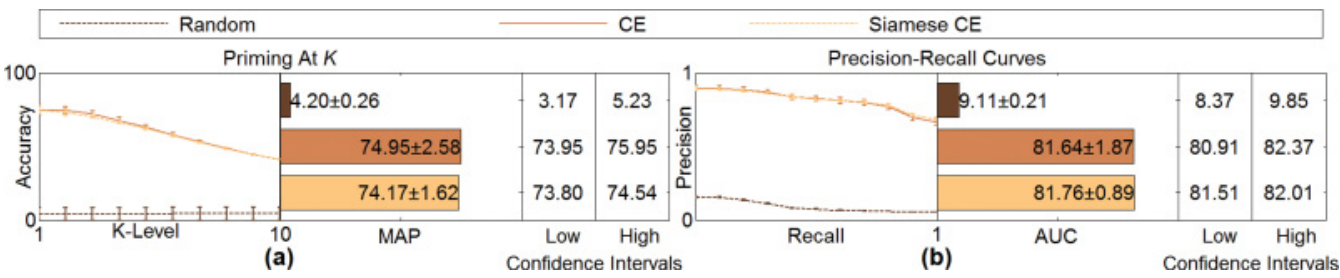

Fig. 7. Semantic priming performance of our model on the OOV tags in MagTag5K.

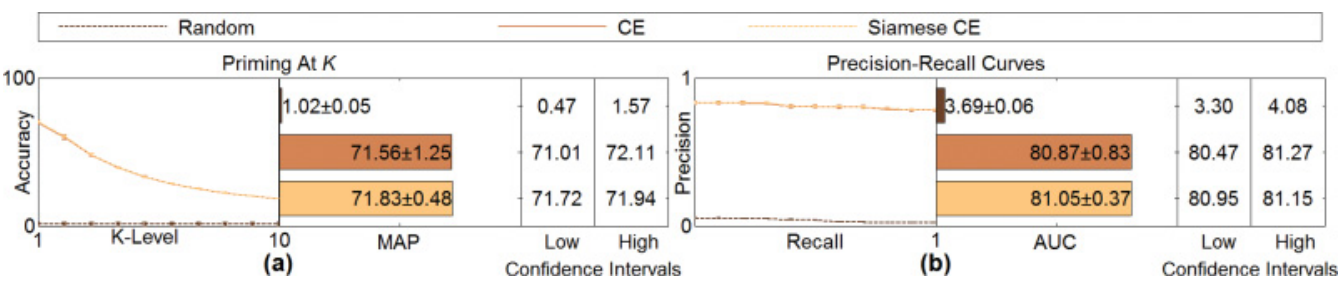

Fig. 8. Semantic priming performance of our model on the OOV tags in MSDSub.

the Random model completely fails. By using the Cohen's d effect size, the difference between Siamese CE and CE is -0.36 in MAP and 0.08 in AUC for in MagTag5K as well as 0.29 in MAP and 0.28 in AUC for MSDSub. In general, the consistent performance has been observed for both datasets.

In summary, the semantics learned by the CE model considerably outperforms that obtained by other state-of-the-art models (even with a nontrivial extension). In particular, the CE model is good at capturing semantics in the sparse BoW scenario and has the unique capability of inferring the semantics from the OOV tags. Hence, we firmly believe that the contextualized semantics learned by the CE model would facilitate various MIR tasks.

\section{TAG COMPLETION}

In this section, we demonstrate the effectiveness of the semantics learned by the CE model in tag completion, a benchmark MIR task that requires suggesting complementary tags to an existing group of tags describing a music track. Likewise, we also compare the $\mathrm{CE}$ model to those models used in the semantic priming evaluation and report comparative results in the tag completion task.

Unlike semantic priming where a query concept is used to identify only its related tags, a query concept in tag completion would result in a score for all tags in descending order in terms of semantic relatedness. Performance evaluation in this task requires the continuous relatedness ground truth that properly reflects semantic coherence among all tags in different contexts. Such information is neither required nor available in the semantic priming task described in Section 3.5. This new information used for evaluation properly differentiates the two tasks. Tag completion evaluation uses only the semantics learned from co-occurring tags. Thus, tag completion becomes an appropriate task to evaluate the quality of semantics learned by the CE model. It is worth stating that the two selected evaluation tasks are not to be confused with Audio Tag Classification task. In the later, music is associated with the relevant tags in one of two modes, binary mode and ranking mode. In both modes, the music content is considered, which is not true for our evaluations. Indeed, semantic priming and tag completion are technically similar as they both operate on the same semantic space, but the difference in nature of the used data results in different evaluation settings. Furthermore, semantic priming, being most generic, is used for early stopping of the CE 
model training (see Section 2.3), while tag completion only measures the performance of such semantics in this specific setting.

\subsection{Dataset}

Continuous track-tag relatedness ground-truth is rarely available for music tag collections as generating such information demands a high level of expertise and is extremely laborious. Fortunately, CAL500 and MSDSub provide the continuous track-tag relatedness ground truth alongside the binary relatedness information [Turnbull et al. 2007; Bertin-Mahieux et al. 2011]. Thus, we employ these two datasets in this evaluation. As a result, each evaluation document from either dataset has two annotation versions, the binary one and the continuous one. In our experiments, we assume that an evaluation document has already been annotated with a group of existing tags based on its binary relatedness version. This assumption is based on the fact that it is impossible to split documents into coherent sets of tags automatically. The evaluation was conducted on the subsets of two datasets, that is, 127 and 3,487 documents in CAL500 and MSDSub, respectively.

\subsection{Evaluation}

Given a tag in local context, all tags are ranked according to the predicted relevance in terms of contextualized semantics learned by a model. This predicted tag ranking, $\boldsymbol{r}$, is evaluated against the continuous relevance ground truth. For evaluation, we employ the Normalized Discounted Cumulative Gain (NDCG) measure [Järvelin and Kekäläinen 2002] across an entire retrieved and ranking list. Given $\boldsymbol{r}[i]$ is the $i^{\text {th }}$ element in $\boldsymbol{r}$, and $\mathrm{rel}(\boldsymbol{r}[i])$ is the ground-truth relevance value of tag $\boldsymbol{r}[i]$; we measure $\mathrm{Dis}$ counted Cumulative Gain (DCG) up to position $k$ as $D C G_{k}(\boldsymbol{r})=\operatorname{rel}(\boldsymbol{r}[1])+\sum_{i=2}^{k} \frac{\operatorname{rel}(\boldsymbol{r}[i])}{\log (i)}$. Similar to P@k, this measure is affected by the length of the document. Therefore, we normalize the DCG by using the best possible DCG for each evaluation document, that is, the list $\boldsymbol{r}_{\text {ideal }}$, the ideal ranking of tags derived from the ground truth given the query tag in context. Thus, the NDCG is measured

$$
N D C G_{k}=\frac{D C G_{k}(\boldsymbol{r})}{D C G_{k}\left(\boldsymbol{r}_{\text {ideal }}\right)},
$$

which assigns higher values to predicted lists with tag ranking closer to the ideal ranking. Changing parameter $k$, we achieve different $\mathrm{NDCG}_{k}$ values. An Averaging NDCG up to $K$ is obtained by averaging all the $\mathrm{NDCG}_{k}$ values for $k \leq K$.

\subsection{Results}

In our experiments, a document with $m$ tags results in $m$ separate queries for evaluation, although they share the same local context. It is worth mentioning that an alternative setting could be aggregating $m$ tags into one query and rank all other tags according to their relatedness to the document query. However, the latter setting does not provide any better insight and may, in fact, cause loss in granularity.

Figure 9 shows the NDCG results on CAL500 including the Averaging NDCG Nalues $_{k}$ at different $k$ levels with the standard error due to the three-fold CV as well as the averaging NDCG value up to $K=20$. All models perform similarly at the small $k$ levels due to the high density underlying this dataset. However, predicting up to 20 reasonable tags appears to be a challenging problem given the fact that the $\mathrm{NDCG}_{k}$ values drop sharply as $k$ increases. Nevertheless, the CE model generally outperforms all others especially for $k>10$, as shown in Figure 9 . The same results are confirmed by the averaging NDCG values up to $K=20$. Overall, Siamese CE performs slightly better than $\mathrm{CE}$ due to the semantic distance learning. 


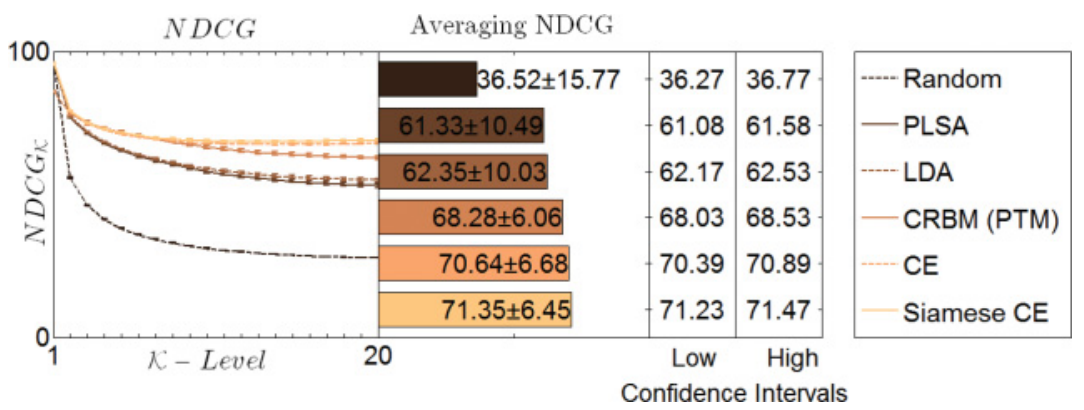

Fig. 9. Normalized Discounted Cumulative Gain evaluation results using CAL500. NDCG values at different $\mathrm{K}$ levels with the standard error as well as the Averaging of NDCG up to 20 levels.

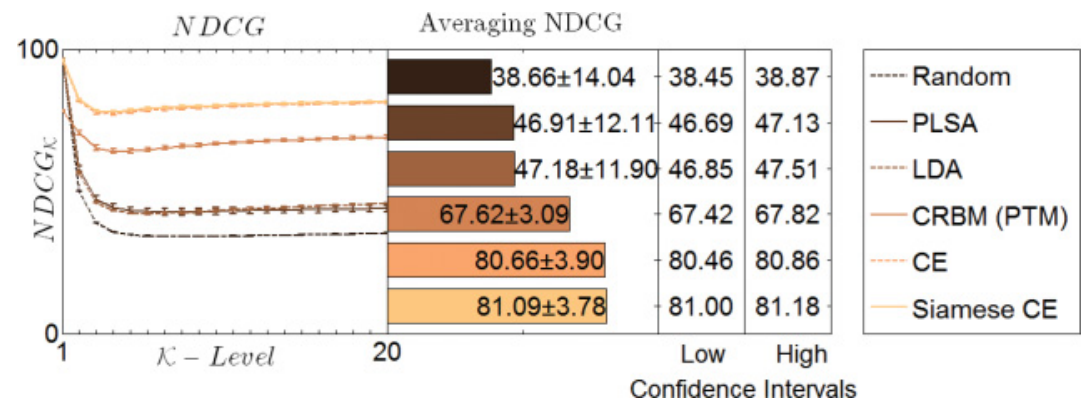

Fig. 10. NDCG performance of different models on MSDSub.

Similarly, Figure 10 shows the NDCG results on MSDSub. Unlike the results on CAL500, the CE model yields the better performance even at small $k$ levels as shown in Figure 10. In MSDSub, the average document length is 3.2. Hence, the performance for $k>3$ would clearly reflect the capability of a model in identifying those reasonable "negative" tags specified in the ground truth. The evidence seen in Figure 10 strongly suggests that the contextualized semantics learned by the CE model leads to better performance with statistical significance (Student's t-test $\mathrm{p}$-value $<0.01$ ).

In summary, the tag completion evaluation further demonstrates the effectiveness of the resultant contextualized semantics learned by the $\mathrm{CE}$ model in capturing the intention of tags' use beyond the co-occurrence statistics. Results reported in two benchmark tasks suggest that the semantics learned by the CE model is ready for use in various MIR applications. It is also worth stating that the CE model may be applied flexibly given the fact that in general, Siamese CE merely outperforms CE slightly on test data but performs considerably better than CE on training data (see the appendix for details). That is, CE would be employed for those MIR tasks that involve many unseen documents in training in order to reduce the training time (c.f. Table I). Otherwise, Siamese CE should be applied for retrieval purposes only.

\section{DISCUSSION}

Unlike previous approaches in semantics learning, the CE model learns distributed semantics without taking any particular MIR tasks into account. This should bring us closer to bridging the semantic gap encountered by various MIR tasks. By considering the local context, our approach leads to distributed multi-representations of a tag associated with different semantic contexts. This salient characteristic distinguishes our approach from others in learning music semantics from tags. 
Previous work in music understanding [Turnbull et al. 2007; Miotto and Lanckriet 2012] focused on performance in one task, namely music annotation or auto-tagging. Indeed, good performance in mapping acoustic content to tags should assist music understanding. However, the resulting models are limited to the training tags only. Moreover, the reliance on acoustic content may result in inconsistent semantics since the contextualizing cues of a tag may have different patterns and are often mixed with other content components. Furthermore, blindly learning to annotate the data by following the given labels may result in biases toward the opinions of the labelers as well as overly fitting any noisy labels in the data [Sturm 2014]. We argue that these limitations can be overcome by using knowledge in the form of semantics learned from crowd-sourced tag collections, as demonstrated in the tag completion task.

In general, expertise-based semantics is transferable across MIR tasks, including (a) attributes listing: the attributes of a tag are manually listed and comparisons are made on the attribute level; (b) ontology: concepts are manually associated [Kim et al. 2008]; and (c) knowledge base: first-order logic rules governing relatedness of tags are maintained [Wang et al. 2010]. Moreover, ontologies have been employed as a categorizing scheme and as a filtering step for tags that happen to be in a dictionary [Cantador et al. 2011]. In comparison to expert-based semantics, CE learned semantics is less interpretable unless additional information is available. Nevertheless, expert-based semantics incurs intensive handcrafted work and suffers from an intrinsic difficulty in quantifying relatedness. These difficulties become more severe in the presence of OOV tags and contexts to consider. In contrast, our approach effortlessly leads to the contextualized semantics and is capable of dealing with OOV tags. Thanks to the limited human intervention in establishing and maintaining CE semantics, which are automatically learned from public tag collections, we firmly believe that it is transferable without biases and, hence, is greatly applicable in various MIR tasks.

Sometimes semantics may be obtained from multiple sources, including artist/track information, users' playlists preferences, tagging information and music production information [Mandel et al. 2011; Weston et al. 2011]. Furthermore, personal intention has been investigated in previous studies [Mika 2005; Mandel et al. 2011]. In such work, the personal meaning of a tag is inferred by analyzing user's tagging activities. Other information sources might include the manual categorization of documents into semantic classes [Font et al. 2014; Font 2015], which allows for better within-class tag similarity estimation once the proper class of a test document is identified. The motivation behind such methods is the construction of rich, transferrable, and often personalized semantics. Aside from the high labor cost, there is no guarantee that the multiple semantics sources are complementary and consistent as integrated semantics. Moreover, finding out possible contradictions between multiple sources may require additional human intervention and more training data. Incomplete and noisy sources further aggravate the semantics fusion. In contrast, our approach does not rely on such information and explores the objective meaning of tags instead. Such objective semantics might easily be used in personalization systems later when coupled with users' information sources or profiles. Thus, we believe that learning semantics from co-occurring tags, a single informative source, is sufficient and justifiable.

Throughout this article, we focused on the contextualized relatedness models. However, global relatedness models have also been used in semantics acquisition due to their simplicity and ease of use. For instance, a specific PCA model is proposed in Lebret et al. [2013] that improved the accuracy of movie review sentiment evaluation. Another successful model is presented in Mandel et al. [2011], where an information theoretic method is used to produce a smoothed representation of a training document which is subsequently applied in music annotation. Other examples include [Mika 2005], where a tag is represented via the information of who used it, and [Font et al. 
2014], where the similarity of tags within each "class" is established via aggregation (c.f. Section 2.1). We acknowledge the usefulness of such models in the music annotation tasks. However, there is evidence that music annotations are contextualized and that meaning cannot be uniquely identified without considering the companion tags as described in Section 1. Similarly, syntactic models learned from large text corpora [Mikolov et al. 2013] yield a single representation for each word. Apart from the fact that these models encode global relatedness only, musical tags are not always in single word form and may contain phrases (e.g., "acoustic guitar"), symbols (e.g., "90s"), and abbreviations (e.g. "r'n'b"). On one hand, it is infeasible to apply these models without domain adaptations into MIR. On the other, adapting such models demands semantics governed by linguistic rules that are absent in tags.

\section{FUTURE WORKS AND CONCLUSIONS}

Exploring intrinsic semantics underlying tags demands a powerful context model that can model the contextual information effectively. PTMs used in the CE model provide a powerful yet generic tool for information aggregation from documents. Unfortunately, such models lack interpretability of the modeled semantics and hence cause the CE model to suffer from the same limitation. For a specific application, however, there is often alternative contextual information and relevant modeling techniques, for example, the labeled LDA [Ramage et al. 2009] trained with meaningful topic labels such as genre or instrument types. In this situation, it is straightforward to incorporate such context representations into the CE model proposed in Section 2. An extension of the CE model by using different context representations may facilitate some MIR tasks that require the self-defined context by users, for example, a playlist of music in a specific style or mood. Moreover, a context modeling the user's behavior can be inferred and issued along with the user's query tags for semantically-coherent query expansion. In fact, the success of the CE model in the Tag completion task hints its suitability for proper query expansion where we explicitly add semantically related keywords to a user's query in order to better describe the user's need in their specific scenario and improve the results of a retrieval task.

Given proper music and/or artist representation within the semantics space, for example, using aggregation of their tags, it becomes straightforward to prime music tracks or artists given user's query concepts. For instance, we can semantically describe a music track as the centroid and the spread statistics of its tags within the semantic space, effectively achieving a semantically descriptive location for each track within the semantic space. As a result, not only a user can query a system for musical content, but also a semantic-level similarity measure between tracks is achieved and can be used for music discovery. Moreover, it becomes easy to annotate music or measure the similarity of artists' work. For example, using a mapping function from the content to the semantic space would facilitate annotating music with known and OOV tags. Such function can also be used to query by example systems.

Finally, the CE model requires the tag features and the BoW output representation. This requirement may prevent the $\mathrm{CE}$ model from being applied to these datasets of a large vocabulary, for example, the complete Million Song Dataset (MSD), due to the high computational burden. There are some technique that could potentially overcome this limitation by generating parsimonious representations, for example, PCA, compressed sensing [Hsu et al. 2009], and filtering techniques [Cantador et al. 2011]. However, such techniques are still under investigation in our ongoing work.

In conclusion, we presented a comprehensive argument for the suitability of contextualized music semantic representations from co-occurring tags in the music domain. The contextualized semantics learned by CE approach significantly outperforms several state-of-the-art semantic learning methods as suggested in our semantic priming 
and tag completion evaluation. Moreover, the semantics learned by the CE model properly deals with the sparse BoW situation as well as the OOV tags. While the work presented in this article is only regarding the contextualized semantic learning from tags and the evaluation was conducted on two generic benchmark tasks, the semantics learned by our approach can be potentially applied to various MIR tasks. As the approach described in this article is a generic approach in learning semantics from any types of tags or descriptive terms regardless of media type, we plan to apply our approach to other domains in our ongoing work.

\section{ACKNOWLEDGMENT}

The authors would like to thank the action editor and anonymous reviewers for their useful comments that improved the manuscript.

\section{REFERENCES}

Yoshua Bengio, Pascal Lamblin, Dan Popovici, and Hugo Larochelle. 2007. Greedy layer-wise training of deep networks. In Advances in Neural Information Processing Systems 19 (NIPS'06). Bernhard Schölkopf, John C. Platt, and Tim Hoffman (Eds.). 153-160.

Thierry Bertin-Mahieux, Douglas Eck, and Michael Mandel. 2010. Automatic tagging of audio: the state-ofthe-art. In Machine Audition: Principles, Algorithms and Systems, Wenwu Wang (Ed.). IGI Publishing, $334-352$.

Thierry Bertin-mahieux, Daniel P. W. Ellis, Brian Whitman, and Paul Lamere. 2011. The million song dataset. In Proceedings of the 12th International Society for Music Information Retrieval Conference (ISMIR'11).

David Blei, Andrew Ng, and Michael I. Jordan. 2003. Latent Dirichlet allocation. J. Mach. Learn. Res. 3 (2003), 993-1022.

Léon Bottou. 2012. Stochastic gradient descent tricks. In Neural Networks, Tricks of the Trade, Reloaded, Grégoire Montavon, Geneviève Orr, and Klaus-Robert Müller (Eds.). Springer-Verlag Berlin Heidelberg, $430-445$.

Jane Bromley, et al. 1993. Signature verification using a "Siamese" time delay neural network. Int. J. Pattern Recognit. Artif. Intell. 7, 4 (August 1993), 669-688.

Iván Cantador, Ioannis Konstas, and Joemon M. Jose. 2011. Categorising social tags to improve folksonomybased recommendations. Web Semant. Sci. Serv. Agents World Wide Web. 9, 1 (March 2011), 1-15.

Jacob Cohen. 1977. Statistical Power Analysis for the Behavioral Sciences Revised Ed., New York, NY, USA.: Academic Press, INC. LTD.

Scott Deerwester, Susan T. Dumais, George W. Furnas, Thomas K. Landauer, and Richard Harshman. 1990. Indexing by latent semantic analysis. J. Am. Soc. Inf. Sci. 41, 6 (1990), 391-407.

Frederic Font. 2015. Tag Recommendation using Folksonomy Information for Online Sound Sharing Platforms. Thesis. Universitat Pompeu Fabra.

Frederic Font, Joan Serrà, and Xavier Serra. 2014. Class-based tag recommendation and user-based evaluation in online audio clip sharing. Knowledge-Based Syst. 67 (2014), 131-142.

Zellig S. Harris. 1954. Distributional structure. WORD-Journal Int. Linguist. Assoc. 10, 2-3 (1954), 146-162.

Thomas Hofmann. 1999. Probabilistic latent semantic indexing. In Proceedings of the 22nd ACM SIGIR Conference on Research and Development in Information Retrieval. New York, NY, USA.: ACM Press, $50-57$.

Daniel Hsu, Sham M. Kakade, John Langford, and Tong Zhang. 2009. Multi-Label prediction via compressed sensing. In Advances in Neural Information Processing Systems 22 (NIPS'09), Yoshua Bengio, Dale Schuurmans, John D. Lafferty, Christopher K. I. Williams, and Aron Culotta (Eds.). 772-780.

Ignacio Iacobacci, Mohammad Taher Pilehvar, and Roberto Navigli. 2015. SensEmbed: Learning sense embeddings for word and relational similarity. In Proceedings of the $53 \mathrm{rd}$ Annual Meeting of the Association for Computational Linguistics (ACL'15). 95-105.

Kalervo Järvelin and Jaana Kekäläinen. 2002. Cumulated gain-based evaluation of IR techniques. ACM Trans. Inf. Syst. 20, 4 (2002), 422-446.

Hak Lae Kim, Simon Scerri, John G. Breslin, Stefan Decker, and Hong Gee Kim. 2008. The state of the art in tag ontologies: A semantic model for tagging and folksonomies. In Dublin Core and Metadata Applications. The Dublin Core Metadata Initiative, 128-137. 
Edith Law, Luis von Ahn, Roger B. Dannenberg, and Mike Crawford. 2007. TagATune: A game for music and sound annotation. In Proceedings of the 8th International Society for Music Information Retrieval Conference (ISMIR'07). 361-364.

Edith Law, Burr Settles, and Tom Mitchell. 2010. Learning to tag from open vocabulary labels. In Proceedings of the 2010 European Conference on Machine Learning and Knowledge Discovery in Databases (EMCL PKDD'10), José L. Balcázar, Francesco Bonchi, Aristides Gionis, and Michèle Sebag (Eds.). Springer, Berlin, 211-226.

Edith Law, Kris West, Michael Mandel, Mert Bay, and J. Stephen Downie. 2009. Evaluation of algorithms using games: The case of music tagging. In Proceedings of the 10th International Society for Music Information Retrieval Conference (ISMIR'09). 387-392.

Rémi Lebret, Joël Legrand, and Ronan Collobert. 2013. Is deep learning really necessary for word embeddings? In Advances in Neural Information Processing Systems 26 (NIPS'13), Deep Learning Workshop.

Mark Levy and Mark Sandler. 2008. Learning latent semantic models for music from social tags. J. New Music Res. 37, 2 (June 2008), 137-150.

Michael S. Lew, Nicu Sebe, Chabane Djeraba, and Ramesh Jain. 2006. Content-based multimedia information retrieval: State of the art and challenges. ACM Trans. Multimed. Comput. Commun. Appl. 2, 1 (2006), $1-19$.

Kevin Lund and Curt Burgess. 1996. Producing high-dimensional semantic spaces from lexical co-occurrence. Behav. Res. Methods, Instruments, Comput. 28, 2 (1996), 203-208.

Laurens van der Maaten and Geoffrey Hinton. 2008. Visualizing data using t-SNE. J. Mach. Learn. Res. 9 (2008), 2579-2605.

Michael I. Mandel, Razvan Pascanu, Douglas Eck, Yoshua Bengio, Luca M. Aiello, Rossano Schifanella, and Filippo Menczer. 2011. Contextual tag inference. ACM Trans. Multimed. Comput. Commun. Appl. 7S, 1, Article 32 (2011), 18.

Christopher D. Manning, Prabhakar Raghavan, and Hinrich Schütze. 2009. An Introduction to Information Retrieval, Cambridge, England: Cambridge University Press, Online edition. Retreived from http://www.informationretrieval.org.

Benjamin Markines, Ciro Cattuto, Filippo Menczer, Dominik Benz, Andreas Hotho, and Gerd Stumme. 2009. Evaluating similarity measures for emergent semantics of social tagging. In Proceedings of the 18th International Conference on World Wide Web (WWW'09). ACM, 641-650.

Gonçalo Marques, Marcos Aurélio Domingues, Thibault Langlois, and Fabien Gouyon. 2011. Three current issues in music autotagging. In Proceedings of the 12th International Society for Music Information Retrieval Conference (ISMIR'11). 795-800.

Peter Mika. 2005. Ontologies Are Us: A Unified Model of Social Networks and Semantics. In The Semantic Web - ISWC 2005, Yolanda Gil, Enrico Motta, V. Richard Benjamins, and Mark A. Musen (Eds.). Springer, Berlin, 522-536.

Tomas Mikolov, Greg Corrado, Kai Chen, and Jeffrey Dean. 2013. Efficient estimation of word representations in vector space. In Proceedings of the International Conference on Learning Representations (ICLR). Scottsdale, AZ, USA.

Riccardo Miotto and Gert Lanckriet. 2012. A generative context model for semantic music annotation and retrieval. IEEE Trans. Audio. Speech. Lang. Processing 20, 4 (2012), 1096-1108.

Rajat Raina, Anand Madhavan, and Andrew Y. Ng. 2009. Large-scale deep unsupervised learning using graphics processors. In Proceedings of the 26th Annual International Conference on Machine Learning (ICML'09). 873-880.

Daniel Ramage, David Hall, Ramesh Nallapati, and Christopher D. Manning. 2009. Labeled LDA: A supervised topic model for credit attribution in multi-labeled corpora. In Proceedings of the 2009 Conference on Empirical Methods in Natural Language Processing: Volume 1, Philip Koehn and Rada Mihalcea (Eds.). ACM, 248-256.

Ubai Sandouk and Ke Chen. 2016. Learning contextualized semantics from co-occurring terms via a Siamese architecture. Neural Networks 76 (April 2016), 65-96.

Markus Schedl, Arthur Flexer, and Julián Urbano. 2013. The neglected user in music information retrieval research. J. Intell. Inf. Syst. 41, 3 (2013), 523-539.

Xavier Serra, Michela Magas, Emmanouil Benetos, Magdalena Chudy, Simon Dixon, Arthur Flexer, Emilia Gómez, Fabien Gouyon, Perfecto Herrera, Sergi Jordà, Oscar Paytuvi, Geoffroy Peeters, Jan Schlüter, Hugues Vinet, and Gerhard Widmer. 2013. Roadmap for Music Information ReSearch (MIReS), Geoffroy Peeters (Ed.). The MIReS Concortium.

Amit Singhal. 2001. Modern information retrieval: A brief overview. IEEE Data Eng. Bull. 24 (2001), 35-43. 
Arnold W. M. Smeulders, Marcel Worring, Simone Santini, Amarnath Gupta, and Ramesh Jain. 2000. Content-based image retrieval at the end of the early years. IEEE Trans. Pattern Anal. Mach. Intell. 22, 12 (2000), 1349-1380.

Mohamed Sordo. 2011. Semantic Annotation of Music Collections: A Computational Approach. Ph.D. Dissertation. Universitat Pompeu Fabra. Barcelona, Spain.

Bob L. Sturm. 2014. The state of the art ten years after a state of the art: Future research in music information retrieval. J. New Music Res. 43, 2 (May 2014), 147-172.

Yee Whye Teh, Michael I. Jordan, Matthew J. Beal, and David M. Blei. 2006. Hierarchical Dirichlet processes. J. Am. Stat. Assoc. 101, 476 (2006).

Douglas Turnbull, Luke Barrington, and Gert Lanckriet. 2008. Five approaches to collecting tags for music. In Proceedings of the 9th International Society for Music Information Retrieval Conference (ISMIR'08).

Douglas Turnbull, Luke Barrington, David Torres, and Gert Lanckriet. 2007. Towards musical query-bysemantic-description using the CAL500 data set. In Proceedings of the 30th Annual International ACM SIGIR Conference on Research and Development in Information Retrieval (SIGIR'07). ACM, 439-446.

Jun Wang, Xavier Anguera, Xiaoou Chen, and Deshun Yang. 2010. Enriching music mood annotation by semantic association reasoning. In Proceedings of the 2010 IEEE International Conference on Multimedia and Expo. IEEE, 1445-1450.

Jason Weston, Samy Bengio, and Philippe Hamel. 2011. Multi-tasking with joint semantic spaces for largescale music annotation and retrieval. J. New Music Res. 40, 4 (2011), 337-348.

Received September 2015; revised May 2016; accepted June 2016 